$42.5 \%$ of the patients. miR-146a and miR-155 showed statistically significant correlation with the diagnosis $\left(r_{s} \quad 0.363\right.$ and 0.330 , respectively) and age $\left(r_{s} \quad 0.239\right.$ and $r_{s} 0.366$, respectively). But none of them correlated with SLEDAI nor with the immunological activity according to ANA, a-dsDNA, a-Sm, a-b2GPI, a-CL antibodies, C3 and C4 complement levels.

Conclusions Our data showed dysregulation of two miRNAs involved in the pathogenesis of SLE by immune cell activation. There was no correlation between the PB levels of these miRNA and the DA as a whole as well as with the immunological activity but larger study is needed to confirm these results.

\section{AUTOPHAGY-RELATED PROTEIN P62 EXPRESSION IS ASSOCIATED WITH CLINICOPATHOLOGIC FEATURES AND PREDNISONE PLUS CTX INDUCTION TREATMENT EFFICACY IN LUPUS NEPHRITIS}

S Sun*, J Chen, Q Yan, C Bao, Q Fu. RenJi Hospital- School of Medicine- Shanghai JiaoTong University, Rheumatology, Shanghai, China

\subsection{6/lupus-2017-000215.318}

Background and aims Previous studies found autophagy contributes to the pathogenesis of systemic lupus erythematosus (SLE). Whether autophagy is involved in lupus nephritis (LN) is not elucidated. P62 is a specific substrate that is degraded through autophagy-lysosomal pathway.

Methods Immunohistochemistry Staining was performed to evaluate expressions of p62 in the biopsy kidney tissue of LN patients $(n=128)$ and normal control $(n=6)$. One hundred and five patients were given prednisone+CTX pulse therapy as induction treatment and followed by 24 weeks. Clinicopathologic features and induction phase remission efficacy were recorded and correlated with renal p62 expression level.

Results Compared with the controls, the expression of p62 was significantly decreased in LN biopsy tissues $(p=0.0013)$, suggesting increased autophagy in LN kidney. Patients with low expression of p62 had less severe nephritis, showing significantly less proteinuria, fewer interstitial fibrosis score and higher estimated creatinine clearance rates $(p=0.0122$, $\mathrm{p}=0.0048, \mathrm{p}=0.0231$, respectively). Logistic regression analysis revealed that lower renal p62 expression was an independent factor associated with $\mathrm{CR}(\mathrm{p}=0.025)$ (Table 1). Patients with low p62 were more likely and quicker to achieve CR (Person Chi-Square test, $p=0.001$; Kaplan-Meier test, $p=0.0294)$.

Conclusions Low renal p62 expression was associated with less severe nephritis and better short-time outcome. Because low p62 expression is the result of high level of autophagy, this data suggested that autophagy might play a protective role in LN kidney. More studies are needed to evaluate the role autophagy plays in multiple organs and cell subtypes in SLE.

\section{ASSOCIATION OF FUNCTIONAL IRF7 VARIANTS WITH SYSTEMIC LUPUS ERYTHEMATOSUS}

J Chen*, C Bao, N Shen, Q Fu. Renji Hospital- Shanghai Jiaotong University School of Medicine, Rheumatology, Shanghai, China

10.1136/lupus-2017-000215.319
Background and aims Previous study identified rs1131665 in IRF7 associated with SLE among multiple ethnic groups. This study was undertaken to investigate whether other genetic polymorphisms within KIAA1542/IRF7 confers risk for the development of SLE.

Methods Four SNPs, including rs4963128, rs702966, rs1131665 (Q412R), rs1061502 (K179E) within KIAA1542/ IRF7 were genotyped in 784 Chinese SLE patients and 899 controls/IRF7 by using Taqman genotyping assay. Luciferase reporter assay, Co-IP and EMSA were used to assess the effect of K179E polymorphism on the activation of IRF7.

Results Q412R and K179E were significantly associated with SLE in Chinese Han population $\left(\mathrm{p}=5.8 \times 10^{-3}, \mathrm{OR}=2.33[1.26-\right.$ 4.33], $\mathrm{p}=2.9 \times 10^{-3}, \mathrm{OR}=2.82[1.38-5.76]$, respectively. IRF7 3'UTR SNP rs702966 was associated with renal involvement $(\mathrm{p}=0.01 \mathrm{OR}=0.46[0.25-0.85]$. Compared with expression of IRF7 179E, expression of IRF7 $179 \mathrm{~K}$ risk allele resulted in a 4-fold increase in ISRE transcriptional activity and stronger ISRE binding activity in EMSA $(\mathrm{p}=0.0002)$, suggesting IRF7 $179 \mathrm{~K}$ confers elevated IRF7 activity. Further study found 179K (lysine) carrying IRF7 protein showed higher acetylation compared to $179 \mathrm{E}$ (glutamic acid) IRF7.

Conclusions We detected a novel association between rs1061502 (K179E) and SLE susceptibility. K179E could change the acetylation of IRF7 in vitro, which might contribute to the transcriptional activity of IRF7.

\section{URINE TWEAK PROTEIN IS A NOVEL BIOMARKER FOR RESISTANT-TO-TREAT LUPUS NEPHRITIS}

${ }^{1,2} \mathrm{~T}_{\text {Suttichet }}{ }^{*}{ }^{3} \mathrm{~T}$ Saetang, ${ }^{4} \mathrm{~S}$ Anutrakulchai, ${ }^{5} \mathrm{~T}$ Panaput, ${ }^{6} \mathrm{~J}$ Wongchinsri, ${ }^{6} \mathrm{~S}$ Chaishayanon, ${ }^{7} \mathrm{~B}$ Satirapoj, ${ }^{8} \mathrm{O}$ Traitanon, ${ }^{9} \mathrm{~B}$ Thinkhamrop, ${ }^{1} \mathrm{Y}$ Avihingsanon. ${ }^{1}$ Faculty of MedicineChulalongkorn University, Department of Medicine, Bangkok, Thailand; ${ }^{2}$ Center of Excellence in Immunology and Immune-mediated diseases- Chulalongkorn University, Department of Microbiology, Bangkok, Thailand; ${ }^{3}$ Systems Biology Center- Faculty of Medicine- Chulalongkorn University, Research Affairs, Bangkok, Thailand; ${ }^{4}$ Faculty of Medicine- Khon Kean University, Department of Medicine, Khon Kean, Thailand; ${ }^{5}$ Khon Kean Regional Hospital, Department of Medicine, Khon Kaen, Thailand; ${ }^{6}$ Nopparat Rajathani Hospital, Department of Medicine, Bangkok, Thailand; ${ }^{7}$ Pramongkutklao School of Medicine, Department of Medicine, Bangkok, Thailand; ${ }^{8}$ Faculty of Medicine- Thammasat University, Department of of Medicine, Pathumthani, Thailand; ${ }^{9}$ Faculty of Public HealthKhon Kean University, Department of Biostatistics and Demography, Khon Kaen, Thailand

\subsection{6/lupus-2017-000215.320}

Background and aims Tumour necrosis factor-like weak inducer of apoptosis (TWEAK) is an inflammatory cytokine that processes via prolonged activation of the NF- $\mathrm{B}$ pathway. TWEAK plays role in autoimmune diseases like lupus nephritis (LN). TWEAK soluble form and its receptor were found in active LN. We determined whether urinary TWEAK (uTWEAK) levels predict response to standard treatment in a multi-centre clinical trial of lupus patients.

Methods Urine samples were collected at baseline, 3 and 6 month of LN patients from a multi-centre randomized-controlled study (Clinicaltrials.gov ID\#NCT01015456). The uTWEAK levels were measured with commercially available enzyme-linked immunosorbent assay (ELISA) kits.

Results All subjects $(n=49)$ were biopsy-proven proliferative class III/IV LN. Median (IQR) patient's age were 32 (29-36) years old. Urine protein creatinine ratio and serum creatinine were $6.32 \quad(6.16-9.20) \mathrm{mg} / \mathrm{mg}$ and $0.80 \quad(0.82-1.03) \mathrm{mg} / \mathrm{dL}$. After 6 month treatment of either intravenous cyclophosphamide (IVCY) or mycophenolate sodium (MPS) and steroids, 
there were 32 responders and 13 non-responders. Baseline uTWEAK levels were higher in non-responder LN compared with responders LN. This was observed in both treatment either IVCY $(n=25)$ or MPS $(n=24)(175.50 \pm 36.97$ vs 57.09 $\pm 7.40 \mathrm{pg} / \mathrm{mL} ; \mathrm{p}=0.018$ or $124.90 \pm 34.53$ vs $55.69 \pm 14.22 \mathrm{pg} /$ $\mathrm{mL} ; \mathrm{p}=0.038)$. The area under the ROC curve to predict response to treatment was $0.79(95 \% \quad \mathrm{CI}=0.64-0.94)$. The cut-off level of $94.0 \mathrm{pg} / \mathrm{dL}$ predict resistant-to-treat at sensitivity and specificity of 64 and 85 percent, respectively.

Conclusions uTWEAK may be a biomarker that guide treatment of lupus nephritis patients. Targeting TWEAK protein in active lupus nephritis is an interesting choice of therapy.

\section{RAPIDLY PROGRESSIVE ILD IN IIM - THE SINGAPOREAN EXPERIENCE}

${ }^{1} \mathrm{TC}$ Tan, ${ }^{2} \mathrm{JWL}$ Tan*, ${ }^{2} \mathrm{KP}$ Leong, ${ }^{2} \mathrm{GYL}$ Chan, ${ }^{2} \mathrm{BYH}$ Thong. ${ }^{1}$ Khoo Teck Puat Hospital, General Medicine, Singapore, Singapore; ${ }^{2}$ Tan Tock Seng Hospital, Rheumatology Allergy and Immunology, Singapore, Singapore

\subsection{6/lupus-2017-000215.321}

Background and aims Interstitial lung disease (ILD) can be associated with the idiopathic inflammatory myopathies (IIM). Rapidly progressive interstitial lung disease (RP-ILD) has been recognised in Asian cohorts and has a high mortality. This study aims to describe a cohort of RP-ILD in Singapore, and identify factors associated with RP-ILD.

Methods This is a retrospective study of IIM-ILD patients in the Department of Rheumatology, Allergy and Immunology, Tan Tock Seng Hospital. Patient symptoms, clinical and serologic features, mortality were compared.

Results There were 68 IIM-ILD patients from 2003-12, 63 cases were analysed. RP-ILD was identified in 14 (22\%). The RP-ILD group was more likely to be male $(42.9 \%$ versus $24.5 \%)$, were more likely to present with fever (50\% vs $14.3 \%, \mathrm{p}=0.01)$, cough $(71.4 \%$ vs $34.7 \%, \mathrm{p}=0.03)$, and dyspnea at rest $(21.4 \%$ vs $2 \%, \mathrm{p}=0.03)$. A larger proportion of patients with RP-ILD had amyopathic dermatomyositis (ADM) (35.7\% vs $20.4 \%)$. None of the patients with RP-ILD had anti-Jo1 (0\% vs 34\%, p=0.01). All RP-ILD cases deteriorated, with $80 \%$ requiring mechanical ventilation. The mortality was $100 \%$ in the RP-ILD group (vs $16 \%$ in non RP-ILD group, $\mathrm{p}=0.00$ ). Median time from diagnosis to death was 26 days. Conclusions We identified ADM and absence of anti Jo-1 as strong associations for RP-ILD. Mortality was $100 \%$ in this RP-ILD cohort. Clinicians should have heightened awareness of this phenotype, early management at specialised respiratory care units, aggressive combination immunosuppressive therapy may be key to mortality reduction.

\section{DOWN-REGULATION OF MIR-10A INDUCES IL-8 IN HUMAN MESANGIAL CELLS STIMULATED WITH ANTI- DSDNA IGG ANTIBODIES}

\footnotetext{
${ }^{1} \mathrm{P}$ Tangtanatakul ${ }^{*},{ }^{2} \mathrm{~B}$ Thammasate, ${ }^{3} \mathrm{~A}$ Jacquet, ${ }^{2} \mathrm{R}$ Reantragoon, ${ }^{4} \mathrm{~T}$ Pisitkun, ${ }^{5} \mathrm{Y}$ Avihingsanon, ${ }^{2} \mathrm{~A}$ Leelahavanichkul, ${ }^{2} \mathrm{~N}$ Hirankarn. ${ }^{1}$ Chulalongkorn University, Microbiology, Bangkok, Thailand; '2Chulalongkorn, Microbiology, Bangkok, Thailand; ${ }^{3}$ Chulalongkorn, Research, Bangkok, Thailand; ${ }^{4}$ Chulalongkorn, Reserach, Bangkok, Thailand; ${ }^{5}$ Chulalongkorn, Medicine, Bangkok, Thailand
}

Background and aims The objective of this study is to investigate the role of miRNA in human mesangial cells (HMCs) stimulated with anti-dsDNA IgG antibodies.

Methods The HMCs were treated with anti-dsDNA IgG antibodies purified from active systemic lupus erythematosus patients or IgG controls in the presence of normal serum for 3 hours. The small RNA expression profile was screened using high throughput sequencing.

Results The results showed that anti-dsDNA IgG up-regulated 103 miRNAs and down-regulated 20 miRNAs which regulate cell cycle, catabolic process, regulation of transcription and apoptosis pathways. Interestingly, miR-10a in HMCs could be validated as specifically down-regulated in HMCs by antidsDNA IgG stimulation. The miR-10a was downregulated in kidney biopsies from lupus nephritis patients and correlated with proteinuria Transiently miR-10a knockdown HMCs increased cells proliferation and up-regulated IL-8 expression. The luciferase assay confirmed that miR-10a down-regulated IL-8 expression by complementary binding to 3'UTR in IL-8.

Conclusions In conclusion, anti-dsDNA IgG Ab down-regulated miR-10a expression in HMC resulting in the induction of various target genes involved in HMCs proliferation as well as inflammation. Manipulation of miR-10a might be a new option for targeted therapy for lupus nephritis.

\section{COMPARISON OF CIRCULATING IMMUNE COMPLEX AND INTERLEUKIN-6 WITH STANDARD BIOMARKERS TO DETERMINE SLE DISEASE ACTIVITY}

${ }^{1} \mathrm{C}$ Thanadetsuntorn*, ${ }^{1} \mathrm{P}$ Ngamjanyaporn, ${ }^{2} \mathrm{C}$ Setthaudom, ${ }^{1} \mathrm{P}$ Pisitkun. ${ }^{1}$ Faculty of Medicine Ramathibodi Hospital, Internal medicine department, Bangkok, Thailand; ' $F a c u l t y$ of Medicine Ramathibodi Hospital, Pathology department, Bangkok, Thailand

\subsection{6/lupus-2017-000215.323}

Background and aims Systemic Lupus Erythematosus (SLE) is an autoimmune disease involving in autoantibody production, immune complex deposition and complement activation. When the disease is active, the sequel can be devastated if inappropriately treated.The outcome of SLE patients can improve if sensitive biomarkers can identify the recent flare and lead the patients to receive the correct treatment in time. This study aimed to investigate whether serum levels of IL-6 and circulating immune complex (CIC) correlated with SLE disease activity and compared with anti-dsDNA and complement.

Methods Ninety SLE patients followed up at Ramathibodi Hospital in 2015 were enrolled. The evaluation of disease activity achieved by Systemic Lupus Erythematosus Disease Activity Index (SLEDAI). The active disease defined if the scores were more than one. Serum IL-6 and CIC tested by ELISA.

Results The level of serum IL-6 and CIC in SLE patients with active disease activity was significantly higher than the inactive

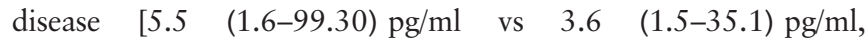
$\mathrm{p}=0.011$ and10.12 (2-131.22) RU/ml vs 2.1 (2.0-101.37) RU/ $\mathrm{ml}, \mathrm{p}=0.011$, respectively]. The correlation analysis between serum biomarkers and clinical SLEDAI demonstrated that biomarkers significantly correlated with SLE activity are CIC $(\mathrm{R}=0.331, \mathrm{p}=0.001)$ and IL-6 $(\mathrm{R}=0.313, \mathrm{p}=0.011)$. CIC had the most area under the curve in discriminating active SLE than IL-6, anti-dsDNA, C4 and C3 (AUC=0.698, 0.677, $0.634,0.410$ and 0.393 respectively) 